\title{
BMJ Evaluation of the filter paper IP-10 tests Open in school children after exposure to tuberculosis: a prospective cohort study with a 4-year follow-up
}

\author{
Tamara Tuuminen, ${ }^{1,2}$ Eeva Salo, ${ }^{3}$ Hannele Kotilainen, ${ }^{4}$ Morten Ruhwald ${ }^{5}$
}

To cite: Tuuminen T, Salo E, Kotilainen $\mathrm{H}$, et al. Evaluation of the filter paper IP-10 tests in school children after exposure to tuberculosis: a prospective cohort study with a 4-year follow-up. BMJ Open 2012;2:e001751. doi:10.1136/bmjopen-2012001751

- Prepublication history for this paper are available online. To view these files please visit the journal online (http://dx.doi.org/10.1136/ bmjopen-2012-001751).

Received 29 June 2012 Accepted 29 October 2012

This final article is available for use under the terms of the Creative Commons Attribution Non-Commercial 2.0 Licence; see http://bmjopen.bmj.com

TT and ES contributed equally to the project and share first authorship.

For numbered affiliations see end of article.

Correspondence to Dr Morten Ruhwald; mruhwald@gmail.com

\section{ABSTRACT}

Objectives: The prevalence of active tuberculosis (TB) is low in Finland, but outbreaks do occur. Following exposure national guidelines recommend either tuberculosis skin test or interferon- $\gamma$-release assaytesting of asymptomatic children. The aim of this study was to compare QuantiFERON-TB Gold In-Tube test (QFT) and interferon- $\gamma$-inducible protein (IP-10) release assay for detection of Mycobacterium tuberculosis infection following exposure to TB in a primary school.

Design: A prospective cohort study.

Setting: School children in Helsinki, Finland.

Participants: Two siblings of the index case and 58 classmates exposed to $M$ tuberculosis.

Intervention: All the children were screened using the QFT, which was used to guide preventive treatment. All those exposed were followed up through the national TB registry.

Outcome measures: IP-10 was measured in plasma supernatants from the QFT test supernatants and in plasma dried and stored for 1 year on filter paper. IP-10 test results were calculated using preset algorithms for positive and indeterminate tests. The negative predictive values of the tests were assessed.

Results: At an initial screening 2 months after the debut of symptoms in the index case, QFT was positive in two children; 56 tests were negative; one was indeterminate and one was borderline. IP-10 showed a perfect concordance between the dried plasma spot and plasma method; two children were IP-10 positive and two were IP-10 indeterminate. There were two $(3 \%)$ discordant results between the QFT and IP-10 tests. Four children converted to positive QFT at a 1-3 month follow-up. None of the QFT negative/borderline children developed TB in the 4-year period since exposure.

Conclusions: We demonstrated that IP-10 and QFT perform comparably as screening tools for infection with $M$ tuberculosis in a contact investigation. IP-10 determined in dried plasma spots was at par with IP-10 determined in plasma, which further supports the usefulness of this alternative approach.

\section{ARTICLE SUMMARY}

\section{Article focus}

- Interferon- $\gamma$ and IP-10 release assays are tests for detection of infection with Mycobacterium tuberculosis, little is known about the performance of IP-10 release assays in contact tracing.

- We have recently developed a novel method for IP-10 detection using dried plasma spots.

- In this study, we compare detection rates and predictive values of IFN- $\gamma$ and IP-10 release assays in a primary school in Finland.

\section{Key messages}

- We demonstrated that IP-10 release assays and the IFN- $\gamma$ release assay (QuantiFERON-TB Gold In-Tube) perform comparably as screening tools in a contact tracing.

- IP-10 determined in dried plasma spots was at par with IP-10 determined in plasma, which further supports the utility of this alternative technology.

Strengths and limitations of this study

- We have used a 4-year follow-up to substantiate our findings.

- Our sample size is small but well characterised and closely monitored.

\section{INTRODUCTION}

Tuberculosis (TB) remains a global epidemic. Childhood TB is a major cause of childhood morbidity and mortality in endemic regions. The diagnosis of TB in children is difficult owing to diverse clinical presentations combined with paucibacillary infection, making bacteriological confirmation challenging. ${ }^{1}$ Children infected with Mycobacterium tuberculosis (latent TB infection (LTBI)) are at a risk of developing TB; wherefore, there is a great benefit from preventive therapy. $^{2}$

Interferon- $\gamma$-release assays (IGRAs) and interferon- $\gamma$-inducible protein (IP-10) release 
assays are immunological tests to identify infection with $M$ tuberculosis. IGRAs are commercially available as the whole blood and ELISA-based QuantiFERON-TB Gold In-Tube test (QFT) (QIAGEN, Valencia, USA), or the PBMC and Eli-SPOT-based T-SPOT.TB test (Oxford Immunotec, Abingdonm UK). IP-10 is a chemokine produced by monocytes following activation by antigenspecific $T$ cells recognising their specific peptide presented on the monocyte surface. IP-10 is released in 100-fold higher concentrations compared to IFN- $\gamma$ making it feasible for detection with a lateral flow, and dried blood and dried plasma spot (DPS) technologies. ${ }^{3}$

A series of clinical studies suggest that IP-10 release assays perform with a comparable sensitivity as IGRAs in patients with a confirmed TB and comparable specificity in unexposed controls, and several studies have found that IP-10 is less affected by low CD4 cell count in HIV-infected individuals and by young age. ${ }^{3-5}$ Little is yet known about the performance of IP-10 release assays for the diagnosis of LTBI following a recent exposure to $M$ tuberculosis in a low-incidence country, nor the predictive values for a positive or negative result.

In this study, we compared QFT to plasma and dried plasma spot-based IP-10 release assays for the detection of LTBI in children, following exposure to TB in a school. We assessed the stability of DPS samples stored for 1 year and determine the negative predictive values (NPV) of the tests in a 4-year follow-up.

\section{MATERIALS AND METHODS \\ Students and setting}

The incidence of TB in Finland is low (6/100 000 inhabitants in 2010) with none to six active cases/year registered among children ( $<15$ years) during the period 2000-2010. ${ }^{6}$

In March 2008, a schoolgirl of African background started to cough, and in June, she was diagnosed with sputum smear-positive TB. As a part of contact tracing the classmates (close contact defined as from 3 to $30 \mathrm{~h}$ / week in the same classroom) and other close contacts were examined for symptoms of active TB and investigated with QFT (June). Siblings were examined for symptoms of active TB and QFT tested at the time of diagnosis of the index case and 1 month later. For both siblings and classmates, positive QFT tests were confirmed with the T-SPOT.TB test. Non-positive QFT tests were repeated 3 months later (September). Four years after the contact tracing, the progression rate in the children was evaluated.

This study was approved by the Ethical Committee of the Hospital for Children and Adolescents, University of Helsinki (Nr.164/E7/05).

\section{IFN- $\gamma$ and IP-10 measurements}

The modified version of QFT was performed according to the standard operating procedure (SOP) adopted in our laboratory (described in detail in refs. ${ }^{7}$ and ${ }^{8}$ ).
In brief, stimulation of blood cells was done in tubes of the manufacturer. However, for measurement of IFN- $\gamma$ levels, we used enzyme immunoassay (EIA) of PeliKine Compact human EIA (Sanquin, Amsterdam, the Netherlands). This results in a steeper calibration curve and a more accurate result interpretation in the cutoff zone. ${ }^{7}$ Thereafter, the excess supernatant from the QFT tubes was stored at $-80^{\circ} \mathrm{C}$ and later shipped on dry ice to the research laboratory at the Copenhagen University Hospital, Hvidovre, Denmark. In August 2010, the samples were thawed and $2 \times 25 \mathrm{\mu l}$ from each sample was pipetted onto Whatman 903 filter paper (Whatman, Maidstone, UK), dried for $4 \mathrm{~h}$ and stored at $-20^{\circ} \mathrm{C}$ for a later analysis; plasma samples were refrozen and stored at $-80^{\circ} \mathrm{C}$. In February 2012, plasma and dried plasma spot samples were analysed for IP-10 using an in-house IP-10 ELISA. ${ }^{9}$

\section{IFN- $\gamma$ and IP-10 test interpretations}

IFN- $\gamma$ and IP-10 concentrations were analysed according to previously set algorithms. For IFN- $\gamma$, two cutoff levels were applied: Samples showing a net reactivity (the reactivity of a sample minus the reactivity of the nil control) $<0.35 \mathrm{IU} / \mathrm{ml}$ were interpreted as negative. Those showing reactivity between 0.35 and $0.50 \mathrm{IU} / \mathrm{ml}$ were interpreted as being borderline and those with a reactivity exceeding $0.50 \mathrm{IU} / \mathrm{ml}$ were interpreted as positive. The IP-10 test was considered positive if net reactivity was above $2.3 \mathrm{ng} / \mathrm{ml}$ (plasma) and $125 \mathrm{pg} / 2 \mathrm{DPS}$ discs and deemed indeterminate if the test was negative and a net reactivity of the positive control was below $1.5 \mathrm{ng}$ (plasma) and $71 \mathrm{pg} / 2$ DPS discs. ${ }^{10}$

\section{Statistics}

Biomarker concentrations were compared using nonparametric statistics. Test results were compared using the McNemars test and $\kappa$ statistics. Correlation was assessed using the Persons method. Data were analysed using the GraphPad Prizm V.5.0 for Mac and SAS V.9.3.

\section{RESULTS}

\section{Children, setting and QFT results}

A total of 67 children were included in the contact investigation; 58 classmates from the school, 7 other close contacts and 2 siblings to the index case (figure 1). Among these seven other close contacts, three had positive QFT and tuberculosis skin test (TST), which were regarded LTBI. These seven samples were not available for IP-10 analysis and they were excluded. A total of 60 individuals were included in the study.

The index case has two siblings. Both were clinically examined and QFT screened at the time of diagnosis of the index case and 1 month later. The sister (age 13) had symptomatic disease at the initial screening in June and received full TB treatment. Her QFT converted from 0 (June) to $18.34 \mathrm{IU} / \mathrm{ml}$ (July). The brother (age 9) was asymptomatic, but received LTBI treatment based on a QFT conversion from 0.0 (June) to $20.4 \mathrm{UI} / \mathrm{ml}$ (August). 


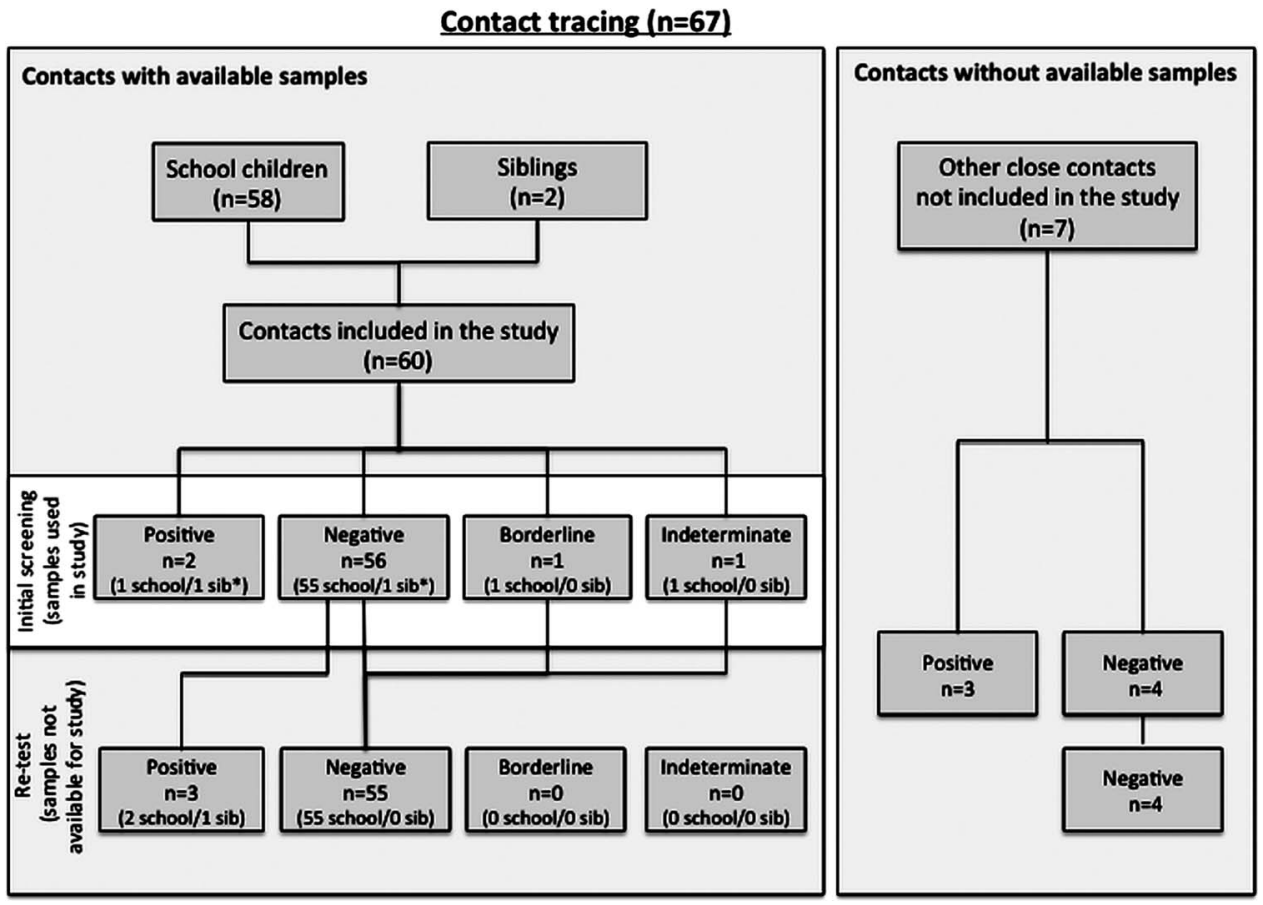

Figure 1 Schematic overview of the contact tracing. Sixty-seven contacts were screened, of which 60 had samples available for the study. Samples from the initial screening (white bar in the middle) were used in the study. ${ }^{*}$ Siblings were screened once before the samples included in this study were obtained. At that time, both tests were negative (see the main text). These samples were not available for the study.

The 58 school children had a median age of 12 years (range 11-14), 40\% (25/58) being male. One child had a missing mitogen sample and was classified as a determinate (valid test). At the initial screening (June), the QFT test identified 1 positive, 1 borderline, 55 negative and 1 indeterminate cases. At a retest, 3 months later two children had converted from no reaction $(0.00$ and $0.02 \mathrm{IU} / \mathrm{ml})$ to strong positive results $(6.24$ and

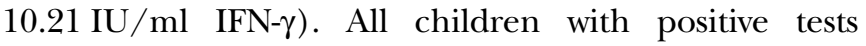
received LTBI treatment.

\section{IP-10 and IFN- $\gamma$ levels}

Nil and antigen samples from the initial screening were available for 58 school children and 1 sibling. The sibling with symptomatic disease was retested 3 weeks after the treatment was started. This sample was available for the study. Raw values for each biomarker are presented in table 1. Correlation between nil, antigen and mitogen samples pooled $(\mathrm{n}=179)$ was very high for IP-10 $\left(r^{2}=0.98\right.$ for IP-10 plasma vs DPS, $\left.\mathrm{p}<0.0001\right)$ and high for IFN- $\gamma$ vs IP-10 $\left(r^{2}=0.69\right.$ and 0.68 for IP-10 plasma and DPS, respectively, $\mathrm{p}<0.0001$, data not shown).

\section{IP-10 test results and concordance}

The two IP-10 tests had a perfect agreement $(60 / 60$, $\mathrm{k}=1.00, \mathrm{p}<0.0001)$ rendering 2 positive, 56 negative and 2 indeterminate (see table 2). The two children who converted to positive QFT at 3 months rescreening were both IP-10 unresponsive at the initial screening. The agreement between QFT and either of the IP-10 tests was $97 \%,(58 / 60, \mathrm{k}=0.86, \mathrm{p}<0.0001$ (borderline classified as negative)). Two children were concordant QFT/IP-10 positive. Both were also positive on T-SPOT.TB used as a confirmatory method.

There were two discordant results. One Finnish-born girl had QFT borderline results $(0.45 \mathrm{IU} / \mathrm{ml} \mathrm{IFN}-\gamma)$; it is noteworthy that she also had the highest spontaneous IFN- $\gamma$ production in the cohort $(0.83 \mathrm{IU} / \mathrm{ml})$. This girl had IP-10 levels of $1.26 \mathrm{ng} / \mathrm{ml}$ and $26 \mathrm{pg} / 2$ DPS spots (plasma and DPS samples, respectively, which is interpreted as IP-10-test negative; figure 2A). She was further tested with T-SPOT.

Table 1 IFN- $\gamma$ and IP-10 levels in plasma and dried plasma spots

\begin{tabular}{llll} 
& Nil & Antigen & Mitogen \\
\hline IFN- $\gamma$ (IU/ml) & $0.04(0.02-0.11)$ & $0.04(0.02-0.12)$ & $9.57(5.52-10.00)$ \\
IP-10 plasma (ng/ml) & $0.16(0.05-0.44)$ & $0.27(0.10-0.53)$ & $9.80(4.84-13.19)$ \\
IP-10 DPS (pg/2 DPS discs) & $4.2(0.00-14.3)$ & $3.3(0.00-14.6)$ & $280.9(148.1-381.2)$ \\
\hline DPS, dried plasma spot; IP-10, interferon- $\gamma$-inducible protein. & &
\end{tabular}


Table 2 Test concordance

\begin{tabular}{|c|c|c|c|c|c|}
\hline & & \multicolumn{3}{|c|}{ IP-10 (plasma or dried plasma spots) } & \\
\hline & & Positive & Negative & Indeterminate & \\
\hline \multirow[t]{5}{*}{ QFT } & Positive & 2 & 0 & 0 & 2 \\
\hline & Borderline & 0 & 1 & 0 & 1 \\
\hline & Negative & 0 & 55 & 1 & 56 \\
\hline & Indeterminate & 0 & 0 & 1 & 1 \\
\hline & & 2 & 56 & 2 & 60 \\
\hline
\end{tabular}

TB and tuberculin skin test, both tests being negative. This child was not offered any treatment.

Another child was IP-10 indeterminate but QFT negative. IFN- $\gamma$ levels in response to mitogen were $1.4 \mathrm{IU} / \mathrm{ml}$, and IP-10 levels were $1.27 \mathrm{ng} / \mathrm{ml}$ and $43 \mathrm{pg} / 2$ DPS discs, in plasma and DPS samples, respectively (figure $2 \mathrm{~B}$ ).

\section{Follow-up}

No new cases were found among the contacts during the following 4 years; the total follow-up time of this study has been 240 person years. None of the seven contacts with a positive test and no symptoms of active TB (four contacts included in the study and the three other close contacts) had recurrent TB.

\section{DISCUSSION}

In this study, we compared the QFT and the IP-10 tests for the diagnosis of LTBI in a cohort of 6th grade school children from an urban setting in Finland. IP-10 was detected in both plasma samples and dried plasma spots and analysed using pre-set test algorithms; the two IP-10 methods were $100 \%$ concordant. Two children were deemed IP-10 and QFT positive. The QFT test showed $97 \%$ agreement with both the IP-10 tests. The children were followed up for 4 years and no new cases were identified, rendering a very high negative predictive value for both QFT and IP-10 tests.

This is the first study to describe the performance of IP-10 in a contact-tracing investigation in school children in a low-endemic setting. We applied IP-10 cutoffs and test algorithms for plasma and DPS estimated in cohorts of cases with active TB and healthy controls. ${ }^{10}$ As expected, IP-10 from dried plasma spots and plasma samples correlated well, and a good agreement between the test results was obtained. ${ }^{9}{ }^{10}$ The DPS samples had been stored in sealed plastic bags with a desiccant for 12 months at $-20^{\circ} \mathrm{C}$ before analysis, apparently without a loss of IP-10 signal. This finding adds to previous experiments of IP-10 stability in dried form on filter paper, where it was shown that there was no loss of IP-10 signal after 4 weeks of storage at temperatures up to $+37^{\circ} \mathrm{C}$. 9

IP-10 and QFT performed similar, albeit 2 of the 60 paired samples were discordant. These findings are in line with previous studies in adults and children without any active TB or comorbidity. ${ }^{11-15}$ One child was QFT borderline/IP-10 negative; this 12-year-old girl was TST and T-SPOT.TB negative at both initial and repeat tests 3 months later. Based on the complete test panel, no treatment was initiated. This child remains asymptomatic. Most probably, this child was not infected and the QFT was false positive, if the interpretation for positivity is taken strictly as instructed. We and others have shown earlier that the QFT assay could have high variation around the cutoff area, thus cautioning not to misinterpret clinical conversions and reversions when sequential samples are analysed. ${ }^{16-18}$ IP-10 is expressed in 100-fold higher levels making detection of low-level responses possible with a simpler technology such as the filter paper assay, but also with a higher accuracy
Figure 2 Correlation plots: (A) Antigen-specific IFN- $\gamma$ vs IP-10 (plasma) release, Lines denotes cutoff for the borderline $0.35-0.50 \mathrm{IU} / \mathrm{ml}$ and positive test $0.50 \mathrm{IU} / \mathrm{ml}$ and $2.3 \mathrm{ng} / \mathrm{ml}$ for IFN- $\gamma$ and $I P-10$, respectively. (B) Mitogen-specific IFN- $\gamma$ vs IP-10 (plasma) release lines denote cutoffs for indeterminate tests $0.5 \mathrm{IU} / \mathrm{ml}$ and $1.5 \mathrm{ng} / \mathrm{ml}$ for IFN- $\gamma$ and IP-10, respectively. Arrows denote discordant responders.
A

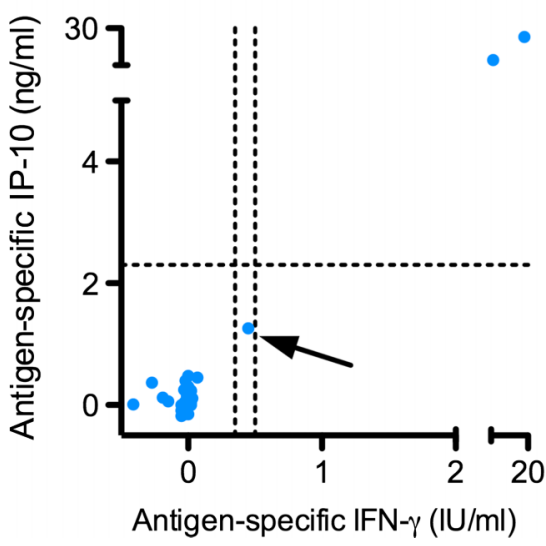

B

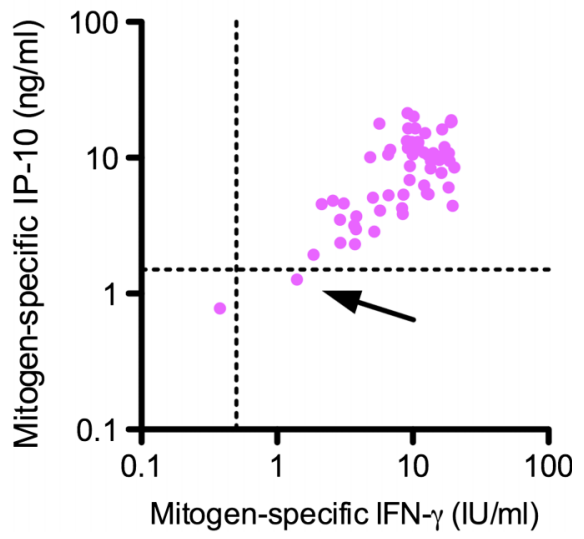


compared to IFN- $\gamma .{ }^{3}{ }^{9}$ Further studies are needed to compare the reproducibility of the IP-10 and QFT ELISAs at concentrations in the range of the diagnostic cutoff.

Another discordant result was in a child who had a negative QFT and an indeterminate IP-10 test result. The IP-10 response to mitogen was only $0.23 \mathrm{ng} / \mathrm{ml}$ below the cutoff for an indeterminate test. ${ }^{10}$ Notably, the current cutoff for indeterminate tests was established on a small set of data, and a larger material is needed to validate this cutoff.

\section{Predictive value}

One of the key questions in the field is how well IGRAs differentiate between people who will develop TB and those who will not, that is, the 'predictive value' of the IGRAs. In this study, none of the 56 children with negative QFT and IP-10 progressed to active TB at a 4 years follow-up, rendering a NPV of $100 \%$. To our knowledge, this is the first assessment of the negative predictive value of IP-10. This finding supports recent reports demonstrating a very high negative predictive value of the IFN- $\gamma$-based IGRAs (reviewed by Diel $e t a l^{19}$ ).

\section{LIMITATIONS}

There is no gold standard for $M$ tuberculosis infection wherefore our assessments of QFT and IP-10 detection rates might be imprecise. Studies in patients with active TB suggest that both tests do not have perfect sensitivity and there is a risk of underestimation of the number of truly infected children. ${ }^{320}$ The long follow-up period brings strength to the assessment of the predictive values, but the low number of observations increases the risk of type-2 error. IFN- $\gamma$ was measured using the SOP developed as HUSLAB. This method ensures a higher degree of accuracy in the IFN- $\gamma$ measurements and has been fully optimised for the QFT interpretation algorithm. ${ }^{717}$ This modified algorithm classifies responses between 0.35 and 0.50 as borderline responses. One child fell into this category, and was subjected to further testing with the T-SPOT.TB and TST. Both these tests as well as the IP-10 test were found to be negative. This finding underlines the importance of interpreting IGRA responses based on both the test result per se and importantly also on the basis of the biomarker concentration.

\section{CONCLUSION}

We demonstrated that IP-10 and QFT perform comparably as screening tools for infection with $M$ tuberculosis in a contact tracing. Both tests demonstrated $100 \%$ NPV. IP-10 determined in dried plasma spots was at par with IP-10 determined in plasma, which further supports the utility of this alternative technology.

\section{Author affiliations}

${ }^{1}$ Department of Bacteriology and Immunology, Haartman Institute, University of Helsinki, Helsinki, Finland
${ }^{2}$ Laboratory of Clinical Microbiology, Eastern Finland Laboratory Centre Joint Authority Enterprise, Mikkeli, Finland

${ }^{3}$ Children's Hospital, University of Helsinki and Helsinki University Central Hospital, Helsinki, Finland

${ }^{4}$ Helsinki Municipal Epidemiology, Helsinki, Finland

${ }^{5}$ Clinical Research Centre, Copenhagen University Hospital, Hvidovre, Denmark

Contributors ES and HK organised the contact investigation, compiled the clinical database and the follow-up of the paediatric TB situation in the area. ES coauthored the manuscript. TT directed the sample collection, IFN- $\gamma$ measurements, compiled the biobank and coauthored the manuscript. MR measured IP-10, analysed the data and wrote the first draft of the manuscript. All the authors participated in data interpretation and all approved the final version of the manuscript.

Funding TT wishes to thank for financial support the following private Finnish foundations: Finnish Lung Health Association (Filha ry), Pulmonary Association Heli and the Tuberculosis Association of the University of Tampere and Nummela Sanatoriums Stiftelse för Medicinsk Forskning. MR is supported by the Danish Foundation for Advanced Technology. The IP-10 ELISA and filter paper method were developed with the kind support from The Danish Lung Association, The Lundbeck-Foundation and The Capital Region of Denmark.

Competing interests Copenhagen University Hospitals, Hvidovre hold the following pending and issued patents disclosing the use of IP-10 as a diagnostic marker for infection with Mycobacterium tuberculosis, MR is registered as an inventor: W02011137902 (filed 04.05.2010) and W02007DK00399 (filed 05.09.2007). This competing interest does not alter the authors' adherence to all the journal policies on sharing data and materials. ES, HK and TT declare no competing interests.

Ethics approval Ethical Committee of the Hospital for Children and Adolescents, University of Helsinki (Nr.164/E7/05).

Provenance and peer review Not commissioned; externally peer reviewed.

Data sharing statement A copy of the dataset is available from the corresponding author at mruhwald@gmail.com. Informed consent for data sharing was not obtained, but the presented data are anonymised wherefore the risk of identification is low.

\section{REFERENCES}

1. Graham SM, Ahmed T, Amanullah F, et al. Evaluation of tuberculosis diagnostics in children: 1. Proposed clinical case definitions for classification of intrathoracic tuberculosis disease. Consensus from an expert panel. J Infect Dis 2012;205(Suppl 2): S199-208

2. Marais BJ, Gie RP, Schaaf HS, et al. The natural history of childhood intra-thoracic tuberculosis: a critical review of literature from the pre-chemotherapy era State of the Art. Int $J$ Tuberc Lung Dis 2004:8:392-402.

3. Ruhwald M, Aabye MG, Ravn P. IP-10 release assays in the diagnosis of tuberculosis infection: current status and future directions. Expert Rev Mol Diagn 2012;12:175-87.

4. Bihari S, Cavalcanti N, Correia JB, et al. Interferon-gamma-inducedprotein-10 concentrations in children with previous tuberculosis infections and disease. Pediatr Infect Dis J 2012;31:1089-91.

5. Yassin MA, Petrucci R, Garie KT, et al. Can interferon-gamma or interferon-gamma-induced-protein-10 differentiate tuberculosis infection and disease in children of high endemic areas? PLoS One 2011;6:e23733.

6. Hulkko T, Lyytikainen O, Jaakola S, et al. Infectious diseases in Finland 2010. National Institute for Health and Welfare (THL) www.thl.fi/thl-client/pdfs/c22be7c1-5e9a-4b2d-a7f8-7be4c530ffe5 (accessed 7 Jun 2012).

7. Tavast E, Salo E, Seppälä I, et al. IGRA tests perform similarly to TST but cause no adverse reactions: pediatric experience in Finland. BMC Res Notes 2009;2:9.

8. Tavast E, Tuuminen T, Pakkanen SH, et al. Immunosuppression adversely affects TST but not IGRAs in patients with psoriasis or inflammatory musculoskeletal diseases. Int $J$ Rheumatol 2012;2012:381929. 
9. Aabye MG, Eugen-Olsen J, Werlinrud AM, et al. A simple method to quantitate IP-10 in dried blood and plasma spots. PLoS One 2012;7: e39228.

10. Aabye MG, Latorre I, Diaz j, et al. Dried plasma spots in the diagnosis of TB: IP-10 release assay on filter paper. Eur Respir J 2012;in press.

11. Ruhwald M, Dominguez J, Latorre I, et al. A multicentre evaluation of the accuracy and performance of IP-10 for the diagnosis of infection with M. tuberculosis. Tuberculosis 2011;91:260-7.

12. Ruhwald M, Bodmer T, Maier C, et al. Evaluating the potential of IP-10 and MCP-2 as biomarkers for the diagnosis of tuberculosis. Eur Respir J 2008;32:1607-15.

13. Frahm M, Goswami ND, Owzar K, et al. Discriminating between latent and active tuberculosis with multiple biomarker responses. Tuberculosis (Edinberg) 2011:91:250-6.

14. Lighter J, Rigaud M, Huie M, et al. Chemokine IP-10: an adjunct marker for latent tuberculosis infection in children. Int $J$ Tuberc Lung Dis 2009;13:731-6.

15. Alsleben $\mathrm{N}$, Ruhwald $\mathrm{M}$, Rüssmann $\mathrm{H}$, et al. Interferon-gamma inducible protein 10 as a biomarker for active tuberculosis and latent tuberculosis infection in children: a case-control study. Scand J Infect Dis 2012;44:256-62.
16. van Zyl-Smit RN, Zwerling A, Dheda K, et al. Within-subject variability of interferon-g assay results for tuberculosis and boosting effect of tuberculin skin testing: a systematic review. PLoS One 2009;4:e8517.

17. Tuuminen $\mathrm{T}$, Tavast $\mathrm{E}$, Vaisanen $\mathrm{R}$, et al. Assessment of imprecision in the interferon gamma release assays (IGRA) for the detection of exposure to Mycobacterium tuberculosis. Clin Vaccine Immunol 2010;17:596-601.

18. Cellestis website. QuantiFERON-TB Gold: precision and reproducibility report_http://www.cellestis.com/IRM/content/gold/ Precisionproductivity.pdf_. 2009. http://www.cellestis.com/IRM/ content/gold/Precisionproductivity.pdf_(accessed 15th November 2012).

19. Diel R, Loddenkemper R, Nienhaus A. Predictive Value of Interferon- $\gamma$ Release Assays and Tuberculin Skin Testing for Progression From Latent TB Infection to Disease State, A Metaanalysis. CHEST J 2012;142:63-75.

20. Diel R, Goletti D, Ferrara G, et al. Interferon- $\gamma$ release assays for the diagnosis of latent Mycobacterium tuberculosis infection: a systematic review and meta-analysis. Eur Respir J 2011; $37: 88-99$ 